\title{
MODELAGEM DO INCREMENTO DIAMÉTRICO PARA ÁRVORES INDIVIDUAIS DE Araucaria angustifolia (BERTOL.) KUNTZE
}

\section{MODELING OF INCREASE DIAMETER FOR INDIVIDUAL TREES OF Araucaria angustifolia (BERTOL.) KUNTZE}

Tatiane Chassot $^{1}$ Frederico Dimas Fleig ${ }^{2}$

\begin{abstract}
RESUMO
Este trabalho teve como objetivo formular modelos de incremento diâmetrico para árvores individuais de Araucaria angustifolia. Foram utilizadas medições de 262 indivíduos de araucária amostrados nas parcelas permanentes do Projeto Ecológico de Longa Duração - "Conservação e Manejo Sustentável de Ecossistemas Florestais - Bioma Araucária e suas Transições" (PELD - CNPq). Utilizando análise de correlação, covariância e regressão, foram ajustados modelos de incremento, estratificados em função da classe de diâmetro, variável de maior correlação com o incremento em diâmetro. Foi utilizado o incremento periódico anual, calculado com base no incremento em diâmetro dos indivíduos no período analisado. Os modelos resultaram em boas estimativas do incremento, no entanto, apresentaram resíduos com forte tendência.
\end{abstract}

Palavras-chave: florestas nativas; crescimento; diâmetro; modelos.

\begin{abstract}
This study aimed to develop diameter increment models for individual trees of Araucaria angustifolia. Were used measurements of 262 araucaria individuals sampled in the permanent plots of the Project PELD - CNPq. Using correlation analysis, covariance and regression, increment models were adjusted, stratified by diameter class, variable with the highest correlation with the increase in diameter. We used the annual periodic increment, calculated based on the diameter increment of the individuals in the analyzed period. Models resulted in good estimates of the increase, however, showed wastes with a strong tendency.
\end{abstract}

Keywords: native forest; growth; diameter; models.

\section{INTRODUÇÃO}

As florestas nativas têm como característica a complexidade em sua composição, ou seja, um grande número de espécies com as mais diferentes características silviculturais, ecológicas e tecnológicas (SCOLFORO et al., 1996).

Estudos em florestas nativas têm sido intensificados nos últimos anos, procurando-se descrever sua composição florística e estrutura fitossociológica, bem como entender a dinâmica desses ecossistemas inequiâneos. No entanto, percebe-se a falta de informações sobre o crescimento individual das espécies dessas florestas.

Uma das maneiras de se obter conhecimentos sobre o crescimento das espécies é por meio de modelos de crescimento. Esses modelos auxiliam as pesquisas e manejo das florestas de várias formas. Um dos importantes usos inclui a possibilidade da predição de produção em tempos futuros, partindo das condições atuais.

1 Engenheira Florestal, Dr ${ }^{\mathrm{a}}$., Professor da Universidade Federal da Fronteira Sul, Av. Jacob Reinaldo Haupenthal, 1580, CEP 97900-000, Cerro Largo (RS), Brasil. tatianechassot@uffs.edu.br

2 Engenheiro Florestal, Dr., Professor do Programa de Pós-Graduação em Engenharia Florestal, Centro de Ciências Rurais, Universidade Federal de Santa Maria, Av. Roraima, 1000, CEP 97105-900, Santa Maria (RS), Brasil. dimasfleig@uol.com.br

Recebido para publicação em 31/07/2014 e aceito em 12/07/2017

Ci. Fl., v. 28, n. 3, jul. - set., 2018 
Em florestas nativas, devido a sua complexidade em número de espécies, com as mais variadas dimensões e distribuição irregular dentro do povoamento, os estudos de crescimento não podem ser realizados com base em valores médios.

Segundo Vanclay (1994), para se determinar o crescimento e incremento de espécies que compõem as florestas inequiâneas e de múltiplas espécies deve-se lançar mão de técnicas de modelagem de crescimento considerando as árvores de forma individual, pois cada espécie apresenta um conjunto de características singulares, como: ritmo de crescimento, necessidades por nutrientes, luz e espaço para crescimento.

De acordo com Vanclay (1991), o desenvolvimento de funções de crescimento para árvores individuais somente terá sucesso, dispondo-se de um grande número de informações e se a espécie estudada ocorrer em grande frequência na área de estudo.

A maioria dos modelos adequados para florestas mistas predizem, explicitamente, o crescimento de árvores individuais, na maioria das vezes, com equações para estimar o crescimento em diâmetro a partir do diâmetro das árvores e outras variáveis. Devido à facilidade de medição, sensibilidade às mudanças ambientais e densidade do povoamento, relação com o tamanho da copa, massa da árvore ou volume do tronco, o diâmetro está diretamente relacionado com o incremento das árvores (ZEIDE, 1989).

Este trabalho teve como objetivo formular modelos de incremento diamétrico para árvores individuais de Araucaria angustifolia, ocorrentes na Floresta Ombrófila Mista da Floresta Nacional de São Francisco de Paula-RS.

\section{MATERIAL E MÉTODOS}

Foram utilizados os dados coletados em 8 unidades amostrais do PELD/CNPq instaladas na Floresta Nacional da São Francisco de Paula, no município de São Francisco de Paula-RS.

A espécie Araucaria angustifolia foi escolhida para este estudo, por se tratar de uma espécie de grande importância, usada principalmente para serraria e laminação, devido à qualidade do fuste e possuir potencial para formar povoamentos homogêneos (REITZ; KLEIN; REIS, 1983; LORENZI, 1998) e também, por ocorrer em grande frequência no local de estudo, apresentando assim um número de dados suficientes para uma estimativa confiável dos resultados obtidos. Para este estudo foram utilizados 262 indivíduos de Araucaria angustifolia.

Foi utilizado o incremento periódico anual, calculado com base no incremento em diâmetro dos indivíduos no período de 8 anos.

\section{Modelagem do incremento}

Para explicar o incremento em diâmetro, procedeu-se a uma análise detalhada das características dimensionais e de concorrência da espécie estudada.

Utilizando-se o software Statistical Package for Social Science for Windows (SPSS 13.0), por meio de análise de correlação, análise de covariância e regressão linear, foi realizada a modelagem do incremento diamétrico das árvores individuais de Araucaria angustifolia.

Para a modelagem foi considerada como variável dependente o incremento em diâmetro e como variáveis independentes, as características dimensionais da espécie e as informações referentes à densidade em torno de cada um dos indivíduos.

As características dimensionais consideradas foram o diâmetro (dap), a altura (h), a relação hd (altura/diâmetro), a posição sociológica (PS), a tendência de valorização (TV) e a classe de copa (CC). Já as variáveis de densidade foram, densidade de $\operatorname{Spurr}\left(\mathrm{G}_{\mathrm{Spurr}}\right)$, densidade de Bitterlich $\left(\mathrm{G}_{\text {Bitterlich }}\right)$ e a porcentagem de araucárias concorrentes.

Os valores de $\mathrm{G}_{\text {Spurr }}$ e $\mathrm{G}_{\text {Bitterlich }}$ foram obtidos utilizando Fator de Área Basal igual a 3. A porcentagem de araucárias concorrentes refere-se à quantidade de $\mathrm{G}_{\text {Spurr }}$ representada somente por Araucaria angustifolia. 


\section{RESULTADOS E DISCUSSÕES}

\section{Variáveis de influência no incremento diamétrico da Araucaria angustifolia}

Apresentaram correlação significativa com o incremento em diâmetro de Araucaria angustifolia as variáveis diâmetro, altura, relação $\mathrm{h} / \mathrm{d}$, posição sociológica, porcentagem de araucárias concorrentes e as densidades de Spurr e de Bitterlich (Tabela 1).

O diâmetro da árvore apresentou correlação positiva com o incremento, demonstrando que árvores com maiores diâmetros têm maiores valores de incremento. Já árvores mais finas, tendem a incrementar menos. Ebling et al. (2012) também observaram as maiores taxas de crescimento nos indivíduos de maiores diâmetros.

A variável de maior correlação com o incremento diamétrico de árvores individuais de Araucaria angustifolia foi a altura da própria árvore, indicando que, quando mais alta for a árvore maior o seu incremento.

A relação altura/diâmetro mostrou efeito negativo sobre o incremento em diâmetro, demonstrando que indivíduos de araucária com uma alta relação $\mathrm{h} / \mathrm{d}$ incrementam menos que indivíduos com relação h/d mais baixa.

Pôde ser observado que a posição sociológica da árvore também é um fator de grande influência no incremento em diâmetro, mostrando que árvores dominantes têm um crescimento maior que árvores dos estratos inferiores. $\mathrm{O}$ valor do coeficiente de correlação apresentou-se negativo, pois no levantamento de dados, as árvores dominantes foram codificadas como sendo igual a 1 , árvores codominantes igual a 2 e as dominadas igual a 3. Dessa forma, quanto maior o número, o qual representa a posição sociológica, menor é o incremento em diâmetro.

TABELA 1: Correlações de Pearson das variáveis dimensionais e de concorrência com o incremento diamétrico de Araucaria angustifolia.

TABLE 1: Pearson correlations of the dimensional variables and competition with the diametric increment of Araucaria angustifolia.

\begin{tabular}{ccccccccccc}
\hline Variáveis & inc & dap & $\mathrm{h}$ & $\mathrm{hd}$ & $\mathrm{PS}$ & $\mathrm{TV}$ & $\mathrm{CC}$ & $\begin{array}{c}\text { Araucárias } \\
\text { concorrentes }\end{array}$ & $\mathrm{G}_{\text {Spurr }}$ & $\mathrm{G}_{\text {Bitterlich }}$ \\
\hline inc & 1 & 0,382 & 0,453 & $-0,397$ & $-0,418$ & 0,007 & $-0,11$ & $-0,278$ & $-0,187$ & $-0,122$ \\
dap & 0 & 1 & 0,791 & $-0,861$ & $-0,732$ & $-0,24$ & $-0,156$ & $-0,183$ & $-0,109$ & $-0,155$ \\
h & 0 & 0 & 1 & $-0,665$ & $-0,747$ & $-0,257$ & $-0,129$ & $-0,067$ & $-0,072$ & $-0,147$ \\
hd & 0 & 0 & 0 & 1 & 0,73 & 0,234 & 0,213 & 0,174 & 0,13 & 0,19 \\
PS & 0 & 0 & 0 & 0 & 1 & 0,154 & 0,161 & 0,169 & 0,109 & 0,121 \\
TV & 0,908 & 0 & 0 & 0 & 0,012 & 1 & 0,003 & $-0,088$ & 0,024 & 0,111 \\
CC & 0,075 & 0,012 & 0,038 & 0,001 & 0,009 & 0,961 & 1 & 0,043 & 0,131 & 0,052 \\
Araucárias & 0 & 0,003 & 0,282 & 0,005 & 0,006 & 0,157 & 0,487 & 1 & $-0,021$ & $-0,006$ \\
concorrentes & & & & & & & & & & \\
G & 0,003 & 0,08 & 0,248 & 0,038 & 0,082 & 0,707 & 0,036 & 0,733 & 1 & 0,492 \\
$\mathrm{G}_{\text {Spurr }}$ & 0,05 & 0,013 & 0,018 & 0,002 & 0,053 & 0,077 & 0,409 & 0,925 & 0 & 1 \\
\hline
\end{tabular}

Em que: inc = incremento em diâmetro, dap: diâmetro a altura do peito, $\mathrm{h}=$ altura total, hd = relação altura diâmetro, $\mathrm{PS}=$ posição sociológica, $\mathrm{TV}=$ tendência de valorização, $\mathrm{CC}=$ classe de copa, araucárias concorrentes $=$ porcentagem de araucárias concorrentes, $\mathrm{G}_{\text {Spurr }}=$ densidade pontual de Spurr, $\mathrm{G}_{\text {Bitterlich }}=$ densidade de Bitterlich. 
Árvores mais altas apresentam a copa livre, não sendo tocadas por nenhuma outra árvore. Devido a isso, uma árvore com a copa livre tem uma grande quantidade de radiação fotossinteticamente ativa incidente diretamente sobre a copa, o que faz com que a produção de fotoassimilados seja grande, refletindo em maiores incrementos.

A tendência de valorização e classe de copa não se mostraram significativas no incremento diamétrico.

A porcentagem de araucárias competidoras mostrou correlação negativa, com índice de correlação igual a - 0,278, sobre o incremento em diâmetro das araucárias centrais. Dessa forma, quanto maior a porcentagem de área basal de araucárias circundantes, menor é o incremento da araucária central.

A densidade de Spurr mostrou efeito negativo sobre o incremento em diâmetro. Quanto maior a densidade de árvores, menor é o incremento em diâmetro. A densidade de Bitterlich também se mostrou significativa, porém, com menor significância.

\section{Análise de covariância e regressão para árvores individuais de Araucaria angustifolia}

Levando-se em consideração a correlação do incremento com o diâmetro e a facilidade de medição deste, foram organizadas classes de diâmetro com amplitude de $10 \mathrm{~cm}$ (Tabela 2).

Em todas as classes pôde ser observado um número expressivo de indivíduos, o que, segundo Vanclay (1991), é imprescindível para estudos de crescimento de árvores individuais. O menor número de indivíduos foi observado na classe de dap 8 , com somente 10 representantes.

TABELA 2: Classes de diâmetro para os indivíduos de Araucaria angustifolia.

TABLE 2: Diameter classes for individuals of Araucaria angustifolia.

\begin{tabular}{ccc}
\hline Classe de diâmetro & Diâmetro $(\mathrm{cm})$ & Número de indivíduos \\
\hline 1 & até 20 & 63 \\
2 & $20-30$ & 42 \\
3 & $30-40$ & 33 \\
4 & $40-50$ & 27 \\
5 & $50-60$ & 39 \\
6 & $60-70$ & 23 \\
7 & $70-80$ & 20 \\
8 & Acima de 80 & 10 \\
\hline
\end{tabular}

Por meio da análise de covariância, foi possível verificar que não houve interação significativa entre as classes de diâmetro com o diâmetro (Classedap*dap) (Tabela 3), indicando que as classes de diâmetro apresentaram a mesma inclinação em relação ao diâmetro das araucárias.

Os coeficientes de cada uma das classes indicaram a semelhança entre as classes de diâmetro. Pôdese verificar que as classes de diâmetro 5, 6,7 e 8 apresentaram valores de coeficiente idênticos, mostrando mesma inclinação das curvas. Para estas classes, os coeficientes angulares não foram significativos, indicando que estes não diferiram da classedap $=8$. 
TABELA 3: Análise de covariância da função inc $=$ dap + classedap*dap para classes de diâmetro para os indivíduos de Araucaria angustifolia.

TABLE 3: Analysis of covariance function inc $=$ dap + classedap*dap to diameter classes for individuals of Araucaria angustifolia.

\begin{tabular}{|c|c|c|c|c|c|}
\hline $\begin{array}{l}\text { Fonte de } \\
\text { variação }\end{array}$ & $\begin{array}{l}\text { Soma de } \\
\text { quadrados }\end{array}$ & $\begin{array}{l}\text { Graus de } \\
\text { liberdade }\end{array}$ & Quadrado médio & $\mathrm{F}$ & Sig. \\
\hline Modelo & 1,696 & 8 & 0,212 & 6,783 & 0 \\
\hline Intercepto & 0,008 & 1 & 0,008 & 0,259 & 0,611 \\
\hline Dap & 0,204 & 1 & 0,204 & 6,533 & 0,011 \\
\hline Classedap*dap & 0,3 & 7 & 0,043 & 1,37 & 0,219 \\
\hline Erro & 7,752 & 248 & 0,031 & & \\
\hline Total & 19,966 & 257 & & & \\
\hline \multicolumn{6}{|c|}{$\mathrm{R}^{2}$ ajustado $=0,153$} \\
\hline Parâmetros & & $\mathrm{B}$ & Erro Padrão & $\mathrm{t}$ & Sig. \\
\hline Intercepto & & $-0,048$ & 0,094 & $-0,509$ & 0,611 \\
\hline dap & & 0,004 & 0,001 & 3,265 & 0,001 \\
\hline$[\text { classedap }=1]^{*}$ dap & & 0,006 & 0,005 & 1,058 & 0,291 \\
\hline$[\text { classedap }=2]^{*}$ dap & & 0,003 & 0,003 & 1,177 & 0,24 \\
\hline$[$ classedap $=3] *$ dap & & 0,004 & 0,002 & 1,936 & 0,054 \\
\hline$[\text { classedap }=4]^{*}$ dap & & 0,003 & 0,001 & 2,275 & 0,024 \\
\hline$[\text { classedap }=5]^{*}$ dap & & 0,001 & 0,001 & 1,131 & 0,259 \\
\hline$[\text { classedap }=6]^{*}$ dap & & 0 & 0,001 & 0,403 & 0,687 \\
\hline$[\text { classedap }=7]^{*}$ dap & & 0,001 & 0,001 & 0,838 & 0,403 \\
\hline$[\text { classedap }=8]^{*}$ dap & & 0 & & & \\
\hline
\end{tabular}

Em que: $\mathrm{F}=$ valor da estatística $\mathrm{F}, \mathrm{B}=$ coeficiente, $\mathrm{t}=$ valor da estatística $\mathrm{t}$, Sig: significância do teste, classedap = classe de diâmetro, dap = diâmetro a altura do peito, $\mathrm{R}^{2}$ ajustado = coeficiente de determinação ajustado.

Procedendo à análise de covariância para verificar a diferença entre as inclinações das demais curvas, verificou-se que a classe de diâmetro 4 apresentou inclinação diferente da classe de diâmetro 5 .

Já as classes de diâmetro 1, 2, 3 e 4 não apresentaram diferença entre as inclinações. Além de não terem sido observadas diferenças entre as inclinações das curvas, também não puderam ser observadas diferenças entre os níveis dessas curvas (Tabela 4). A variável classe de dap não se mostrou significativa. 
TABELA 4: Análise de covariância da função inc $=$ dap + classedap para classes de diâmetro para os indivíduos de Araucaria angustifolia.

TABLE 4: Analysis of covariance function inc $=$ dap + classedap to diameter classes for individuals of Araucaria angustifolia.

\begin{tabular}{|c|c|c|c|c|c|}
\hline $\begin{array}{l}\text { Fonte de } \\
\text { variação }\end{array}$ & $\begin{array}{c}\text { Soma de } \\
\text { quadrados }\end{array}$ & $\begin{array}{l}\text { Graus de } \\
\text { liberdade }\end{array}$ & $\begin{array}{l}\text { Quadrado } \\
\text { médio }\end{array}$ & $\mathrm{F}$ & Sig \\
\hline Modelo & 1,755 & 8 & 0,219 & 7,072 & 0 \\
\hline Intercepto & 0,085 & 1 & 0,085 & 2,752 & 0,098 \\
\hline Dap & 0,255 & 1 & 0,255 & 8,213 & 0,005 \\
\hline Classedap & 0,358 & 7 & 0,051 & 1,651 & 0,122 \\
\hline Erro & 7,694 & 248 & 0,031 & & \\
\hline Total & 19,966 & 257 & & & \\
\hline \multicolumn{6}{|c|}{$\mathrm{R}^{2}$ ajustado $=0,159$} \\
\hline \multicolumn{2}{|c|}{ Parâmetros } & $\mathrm{B}$ & Erro Padrão & $\mathrm{t}$ & Sig. \\
\hline \multicolumn{2}{|c|}{ Intercepto } & $-0,637$ & 0,335 & $-1,901$ & 0,058 \\
\hline \multicolumn{2}{|c|}{ dap } & 0,011 & 0,004 & 2,866 & 0,005 \\
\hline \multicolumn{2}{|c|}{$[$ classedap=1] } & 0,571 & 0,28 & 2,044 & 0,042 \\
\hline \multicolumn{2}{|c|}{ [classedap $=2]$} & 0,503 & 0,242 & 2,083 & 0,038 \\
\hline \multicolumn{2}{|c|}{$[$ classedap $=3]$} & 0,483 & 0,209 & 2,312 & 0,022 \\
\hline \multicolumn{2}{|c|}{$[$ classedap $=4]$} & 0,41 & 0,166 & 2,47 & 0,014 \\
\hline \multicolumn{2}{|c|}{$[$ classedap $=5]$} & 0,271 & 0,132 & 2,048 & 0,042 \\
\hline \multicolumn{2}{|c|}{ [classedap $=6]$} & 0,159 & 0,102 & 1,563 & 0,119 \\
\hline \multicolumn{2}{|c|}{$[$ classedap $=7]$} & 0,121 & 0,079 & 1,527 & 0,128 \\
\hline \multicolumn{2}{|c|}{$[$ classedap $=8]$} & 0 & . & . & . \\
\hline
\end{tabular}

Em que: $\mathrm{F}=$ valor da estatística $\mathrm{F}, \mathrm{B}=$ coeficiente, $\mathrm{t}=$ valor da estatística $\mathrm{t}$, Sig: significância do teste, classedap $=$ classe de diâmetro, dap = diâmetro a altura do peito, $\mathrm{R}^{2}$ ajustado = coeficiente de determinação ajustado.

Entretanto, assim como observado para as inclinações, para a diferença dos níveis das curvas, algumas classes de diâmetro mostraram coeficientes significativos.

Dessa forma, procedeu-se à análise dos dados, desconsiderando a possibilidade de agrupamento das classes de diâmetro.

Para cada classe de diâmetro foram realizadas tentativas de ajuste de um modelo de regressão. No entanto, as tentativas de modelagem do incremento, apesar de bom ajuste em termos de coeficiente de determinação ajustado, resultaram em modelos com alto erro padrão da estimativa (Tabela 5) e com resíduos relativamente altos e fortemente tendenciosos (Figura 1). 
TABELA 5: Modelos de incremento para árvores individuais de Araucaria angustifolia.

TABLE 5: Increment models for individual trees of Araucaria angustifolia.

\begin{tabular}{c|c|c|c}
\hline Classes & Modelo & $\mathrm{R}^{2}$ ajust & Syx $\%$ \\
\hline 1 & $1,263+0,104 * \mathrm{~h}-1,383 * \mathrm{hd}-0,091 *$ dap & 0,697 & 70,79 \\
2 & $0,015 * \mathrm{~h}-0,002 *$ porcentagem de araucárias concorrentes & 0,599 & 86,23 \\
3 & $0,095 * \mathrm{~h}-2,257 * \mathrm{hd}-0,008 * \mathrm{G}_{\text {Spurr }}$ & 0,742 & 69,14 \\
4 & $0,057 * \mathrm{~h}-0,003 *$ porcentagem de araucárias $-1,750 * \mathrm{hd}$ & 0,762 & 60,51 \\
5 & $0,701-0,003 *$ porcentagem de araucárias $-0,007 * \mathrm{G}_{\mathrm{Spurr}}$ & 0,263 & 72,97 \\
6 & $-1,624+1,883 * \mathrm{hd}+0,019 *$ dap & 0,296 & 47,77 \\
7 & $-0,797-0,004 *$ porcentagem de araucárias $+0,061 * \mathrm{~h}$ & 0,593 & 52,15 \\
8 & $0,013 * \mathrm{~h}$ & 0,79 & 51,94 \\
\hline
\end{tabular}

Em que: $\mathrm{h}=$ altura total, $\mathrm{hd}=$ relação altura diâmetro, dap = diâmetro a altura do peito, $\mathrm{G}_{\mathrm{Spurr}}=$ densidade pontual de Spurr, $\mathrm{R}^{2}$ ajust = coeficiente de determinação ajustado, Syx\% = erro padrão da estimativa em percentagem.

Stepka et al. (2012), utilizando estratificações dos dados, testaram vários modelos para estimar o incremento em diâmetro de Araucaria angustifolia em uma Floresta Ombrófila Mista no centro-sul do Paraná. Os modelos não foram eficientes na predição do incremento por apresentarem baixíssimo ajuste, considerando o coeficiente de determinação, e altos valores do erro padrão da estimativa.

Como os modelos gerados não se mostraram eficientes na predição do crescimento dos indivíduos de Araucaria angustifolia, foram analisadas as características desses indivíduos considerando-se as classes de diâmetro. Para essas análises, foi apontado como expressivo o crescimento de, no mínimo, $0,2 \mathrm{~cm} /$ ano.

Levando em consideração a forte correlação da altura com o diâmetro (Tabela 1), foram graficadas essas duas variáveis nas quais se pôde observar que os indivíduos de araucária apresentaram valores de altura distintos para cada classe de diâmetro (Figura 2). Para cada classe, os valores de altura ocuparam diferentes posições no gráfico. 

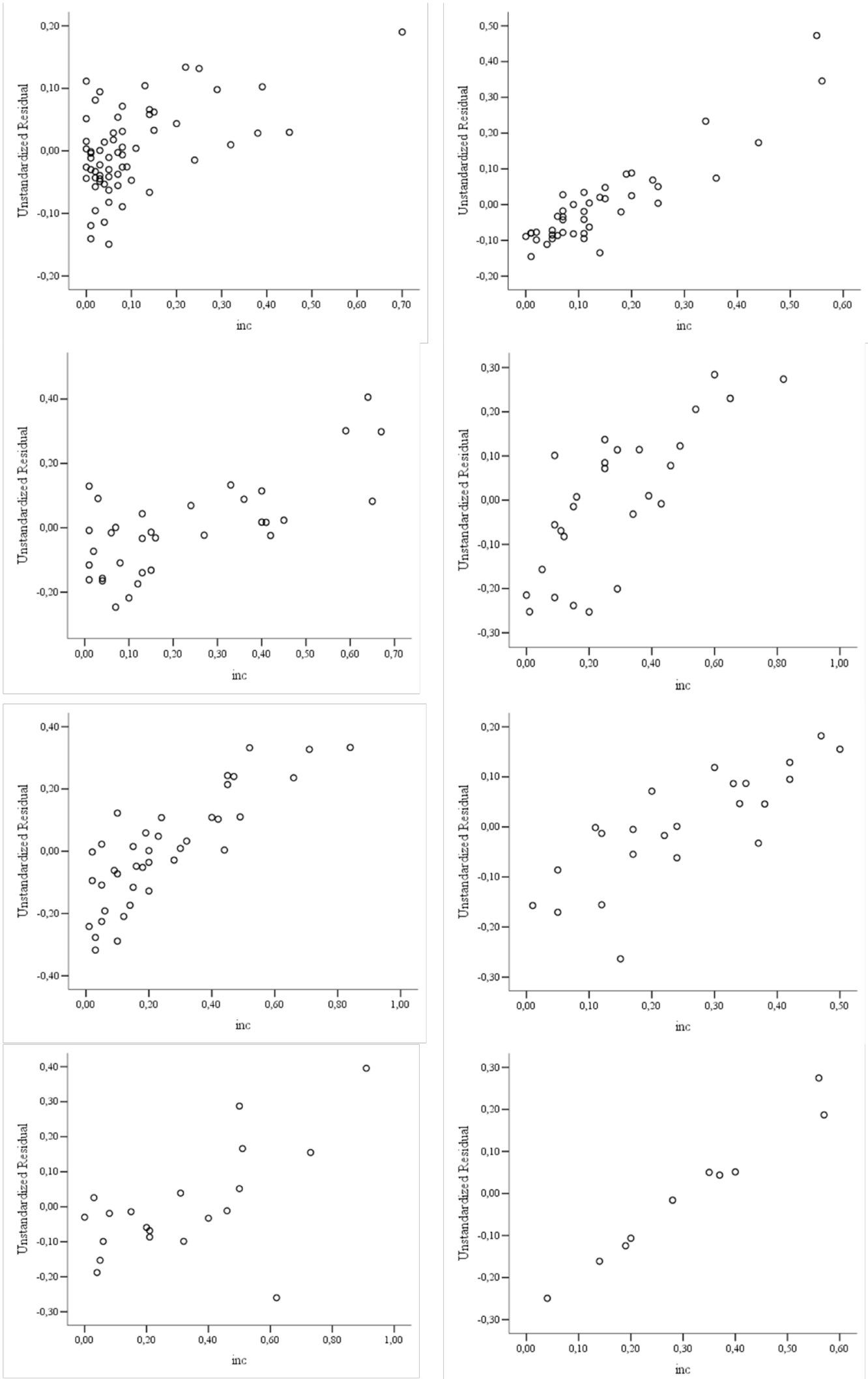

FIGURA 1: Resíduos dos modelos de regressão para árvores individuais de Araucaria angustifolia. FIGURE 1: Waste of regression models for individual trees of Araucaria angustifolia. 


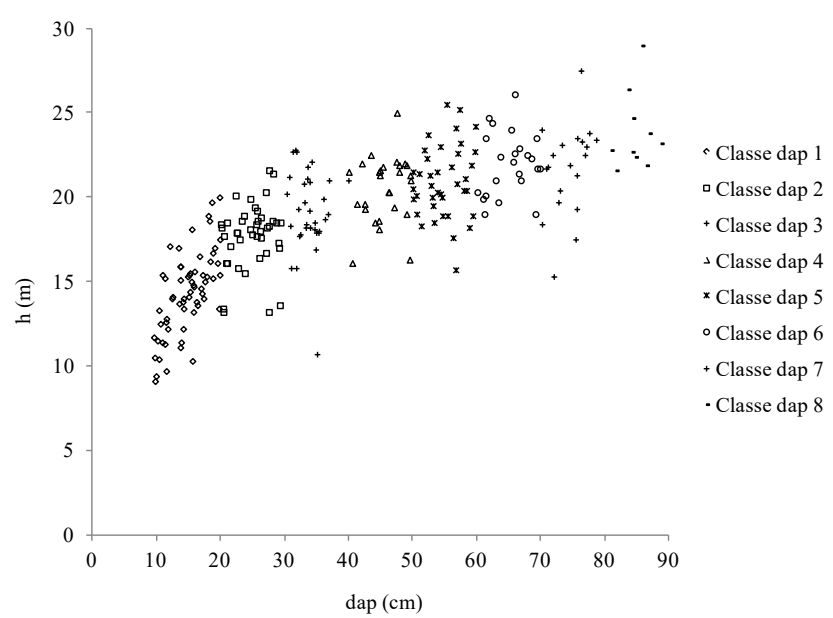

FIGURA 2: Valores de altura dos indivíduos de Araucaria angustifolia em relação às classes de diâmetro.

FIGURE 2: Height values of individuals of Araucaria angustifolia in relation to the diameter classes.

Indivíduos da classe de dap 1 apresentaram altura variando de 9 a $20 \mathrm{~m}$; representantes da classe de dap 2 variaram sua altura entre 13 e $22 \mathrm{~m}$; na classe de dap 3, a altura variou de 15 a $23 \mathrm{~m}$, sendo um indivíduo com altura de, aproximadamente, $11 \mathrm{~m}$, o qual foi classificado como codominante (PS 2); para indivíduos da classe de dap 4, a altura apresentou variação de 16 a $25 \mathrm{~m}$; indivíduos da classe de dap 5 com amplitude de 16 a 26 m na altura; indivíduos da classe de dap 6 apresentaram variação de 19 a 26 m; para a classe de dap 7 foi observada uma maior dispersão dos valores de altura, não seguindo a tendência das demais classes, sendo que os valores de altura variaram de 15 a 28 m e esta maior variação foi decorrente de três árvores que apresentaram valores de altura inferior a $19 \mathrm{~m}$ (limite inferior da classe anterior); a classe de dap 8, teve variação de altura entre 21 e $29 \mathrm{~m}$.

\section{Classes de diâmetro 1}

A distribuição dos incrementos em diâmetro em relação ao dap para os indivíduos de Araucaria angustifolia com diâmetro menor que $20 \mathrm{~cm}$ se mostrou sem tendência, sendo observado qualquer valor de incremento para cada um dos valores de diâmetro (Figura 3). Além disso, indivíduos com crescimento de até $0,2 \mathrm{~cm} /$ ano foram observados para a maioria dos valores de diâmetro.

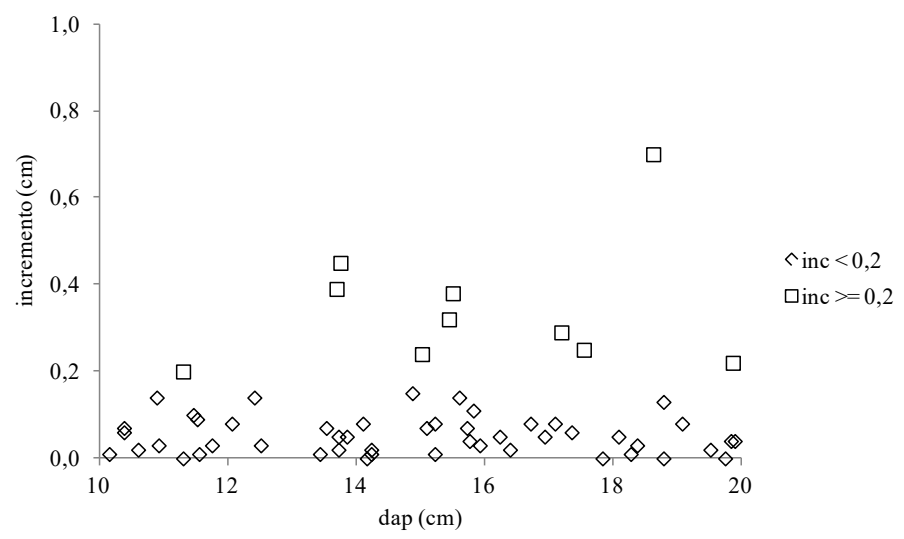

FIGURA 3: Distribuição dos incrementos em diâmetro em relação ao dap para os indivíduos de Araucaria angustifolia com diâmetro menor que $20 \mathrm{~cm}$.

FIGURE 3: Distribution of increments in diameter compared to dap for individuals of Araucaria angustifolia with less than $20 \mathrm{~cm}$ diameter.

Considerando-se a correlação significativa da altura com o incremento em diâmetro dos indivíduos de Araucaria angustifolia (Tabela 1) e a relação da altura com o diâmetro para cada classe de dap observada 
na Figura 2, foi estudada a influência da altura sobre o incremento em diâmetro (Figura 4).

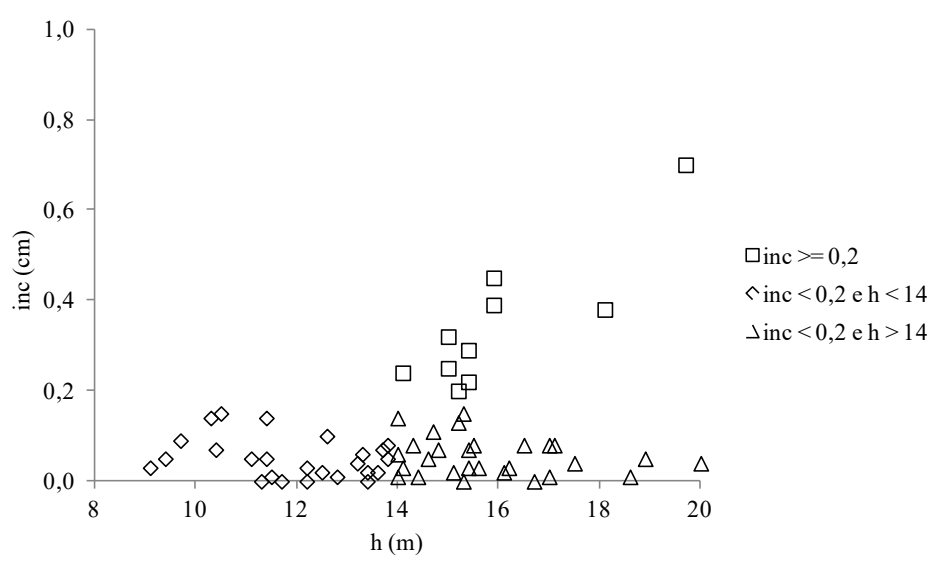

FIGURA 4: Distribuição dos incrementos em diâmetro em relação à altura para os indivíduos de Araucaria angustifolia com diâmetro menor que $20 \mathrm{~cm}$.

FIGURE 4: Distribution of increments in diameter in relation to the height of Araucaria angustifolia individuals with less than $20 \mathrm{~cm}$ diameter.

A altura mostrou-se um fator determinante para o crescimento desses indivíduos de Araucaria angustifolia. Foi observado que todos os indivíduos com crescimento maior que $0,2 \mathrm{~cm} /$ ano apresentaram altura superior a $14 \mathrm{~m}$.

Dessa forma, indivíduos de Araucaria angustifolia, com diâmetro menor que $20 \mathrm{~cm}$, somente apresentarão crescimento considerável quando possuírem uma altura superior a $14 \mathrm{~m}$.

Ainda para as árvores com crescimento superior a $0,2 \mathrm{~cm} / \mathrm{ano}$, observou-se também um maior crescimento para aquelas de maior altura. Indivíduos que incrementaram de 0,2 a $0,4 \mathrm{~cm} /$ ano apresentaram altura entre 14 e $16 \mathrm{~m}$. Já os indivíduos com os maiores valores de incremento foram árvores com mais de $16 \mathrm{~m}$ de altura.

Os indivíduos dessa classe de diâmetro com altura menor que 14 m não alcançaram incremento considerável. Pôde-se observar que todos os indivíduos com essa dimensão de altura incrementaram menos que $0,2 \mathrm{~cm} /$ ano.

No entanto, para os indivíduos com altura maior que $14 \mathrm{~m}$, também foram observados incrementos menores que $0,2 \mathrm{~cm} /$ ano. Para esses indivíduos com altura maior que $14 \mathrm{~m}$, procedeu-se a uma análise mais detalhada, considerando a densidade de Spurr e a porcentagem de araucárias circundantes à árvore central (Figura 5).

A maioria dos indivíduos com incremento menor que $0,2 \mathrm{~cm} /$ ano (Figura $5 \mathrm{~B}$ ) e altura maior que $14 \mathrm{~m}$ apresentou valores de porcentagem de araucárias circundantes superior a $40 \%$, o que indicou que, aproximadamente, mais da metade das árvores concorrentes de cada uma das araucárias centrais foram também araucárias. A grande quantidade de araucárias circundantes à araucária central exerceu efeito negativo sobre o crescimento.

Além disso, esses indivíduos também apresentaram altos valores de densidade de Spurr, exceto um indivíduo que apresentou densidade de $28 \mathrm{~m}^{2} / \mathrm{ha}$, outro com $32 \mathrm{~m}^{2} / \mathrm{ha}$ e dois indivíduos com $33 \mathrm{~m}^{2} / \mathrm{ha}$.

A maioria dos indivíduos com crescimento reduzido apresentou forte competição. No entanto, algumas árvores de araucárias, mesmo estando sob baixa porcentagem de araucárias concorrentes, com valores abaixo de $20 \%$, não apresentaram incremento considerável. Contudo, essas árvores apresentaram altos valores da densidade de Spurr.

Apesar dos indivíduos de Araucaria angustifolia estarem em condições de baixa porcentagem de araucárias concorrentes, a alta densidade de Spurr influenciou negativamente o incremento em diâmetro. Assim, a densidade de Spurr mostrou ser uma variável de forte influência sobre o incremento em diâmetro, independentemente de ser constituída por folhosas ou araucárias circundantes.

No entanto, os indivíduos de Araucaria angustifolia com crescimento superior a 0,2 cm/ano (Figura 
5A) também apresentaram altos valores de densidade de Spurr. Somente três indivíduos estiveram sob densidade menor ou igual a $33 \mathrm{~m}^{2} / \mathrm{ha}$.

Apesar da alta densidade, os indivíduos de araucária encontraram-se em situações de baixa porcentagem de araucárias concorrentes, com valores abaixo de $40 \%$. Nesse sentido, entendeu-se que, a alta densidade foi formada por árvores folhosas e esta não afetou o incremento em diâmetro. Além disso, a altura superior a $14 \mathrm{~m}$ favoreceu o crescimento desses indivíduos.

Indivíduos de Araucaria angustifolia com altura maior que $14 \mathrm{~m}$ circundados por espécies folhosas, mesmo em alta densidade, apresentaram potencial de crescimento superior a $0,2 \mathrm{~cm} /$ ano.

Dois indivíduos de Araucaria angustifolia apresentaram incremento superior a 0,2 cm/ano mesmo estando em condições de alta porcentagem de araucárias circundantes. Porém, a densidade sob a qual estes indivíduos estiveram submetidos não foi tão alta, sendo os valores de 33 e $39 \mathrm{~m}^{2} / \mathrm{ha}$. Nesse caso, entendeu-se que esta densidade foi formada a partir de um grande número de araucárias circundantes, mas de pequenas dimensões.

De maneira geral, altos valores da densidade de Spurr e da porcentagem de araucárias circundantes influenciam negativamente o incremento em diâmetro dos indivíduos de araucária.

No entanto, indivíduos com altos valores de densidade de Spurr apresentarão crescimento significativo quando esta densidade for formada por espécies folhosas, ou seja, a araucária central estiver circundada por outras espécies que não a araucária.

Além disso, os indivíduos de araucária igualmente apresentarão crescimento considerável quando estiverem circundados por um grande número de araucárias, desde que estas tenham baixas dimensões, resultando em baixos valores de densidade de Spurr.

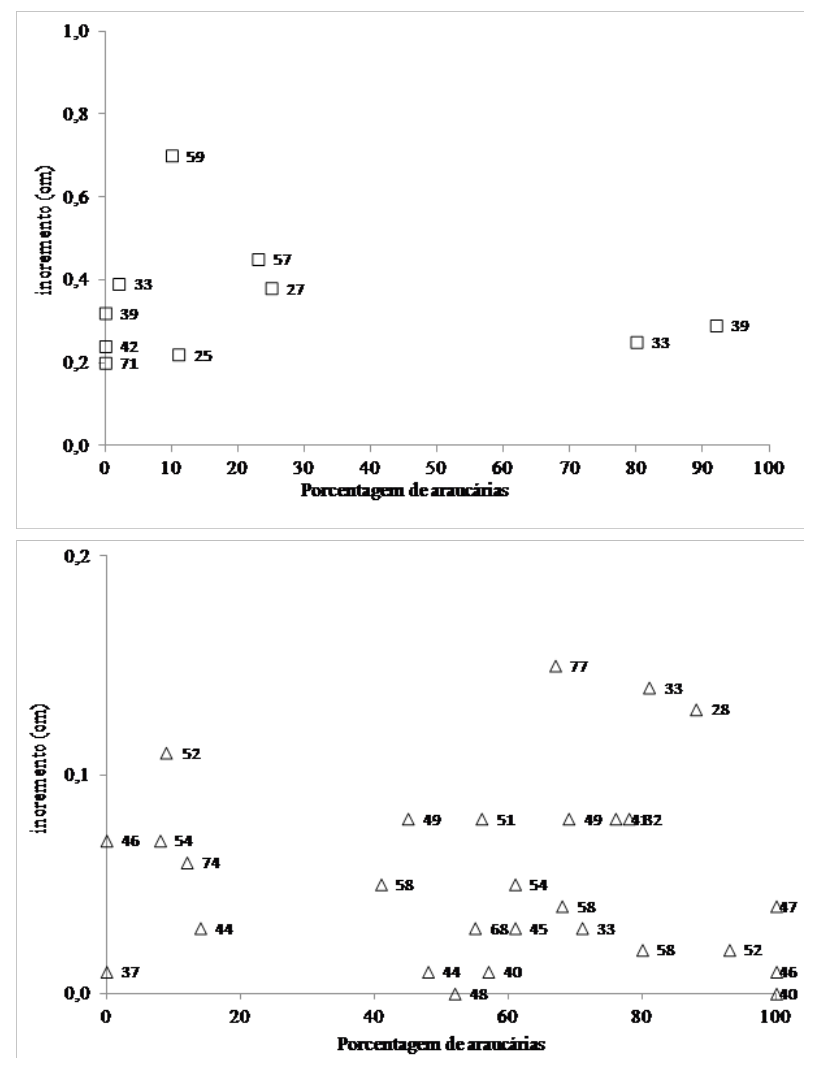

FIGURA 5: Distribuição dos incrementos em diâmetro em relação à porcentagem de araucárias concorrentes para os indivíduos de Araucaria angustifolia da classe de diâmetro 1 (Obs: os valores do rótulo de dados representam a densidade pontual de Spurr).

FIGURE 5: Distribution of increments in diameter compared the percentage of competitors araucarias for individuals of Araucaria angustifolia of the diameter class 1. 


\section{Classes de diâmetro 2}

Assim como observado para os indivíduos da classe de dap 1, para os indivíduos com diâmetro entre 20 e $30 \mathrm{~cm}$, a distribuição dos incrementos em diâmetro em relação ao dap também se mostrou sem tendência, sendo novamente observado qualquer valor de incremento para cada um dos valores de diâmetro (Figura 6).

Indivíduos com crescimento de até $0,2 \mathrm{~cm} /$ ano puderam ser observados para todos os valores de diâmetro e, igualmente foram considerados como árvores com crescimento reduzido e estudados separadamente.

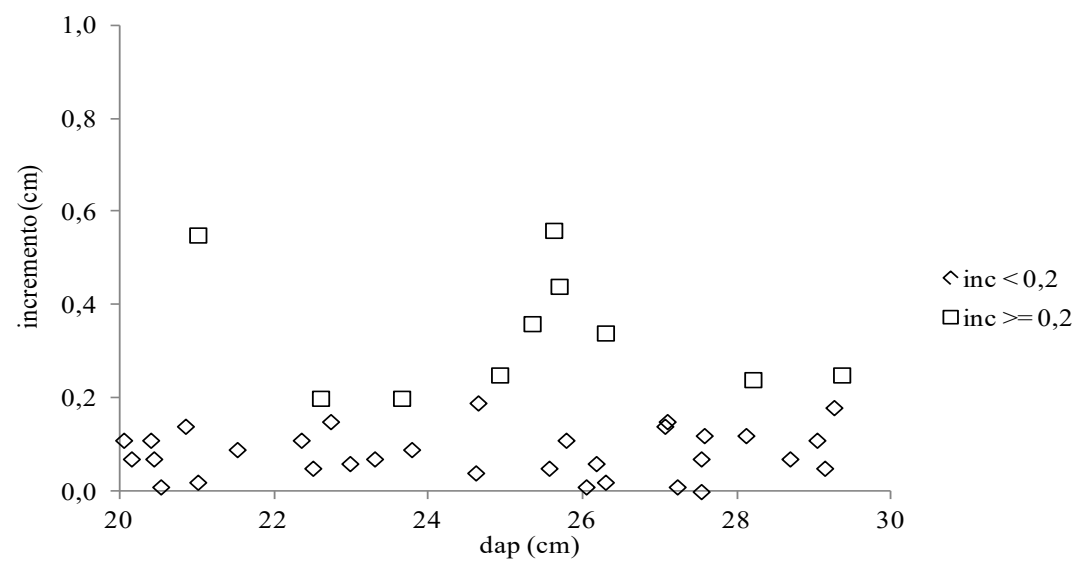

FIGURA 6: Distribuição dos incrementos em diâmetro em relação ao dap para os indivíduos de Araucaria angustifolia com diâmetro entre 20 e $30 \mathrm{~cm}$.

FIGURE 6: Distribution of increments in diameter compared to dap for individuals of Araucaria angustifolia with diameters between 20 and $30 \mathrm{~cm}$.

Para esta classe de diâmetro, a altura também exerceu influência sobre o incremento em diâmetro (Figura 7). Todos os indivíduos com crescimento superior a $0,2 \mathrm{~cm} /$ ano apresentaram altura superior a 16 $\mathrm{m}$.

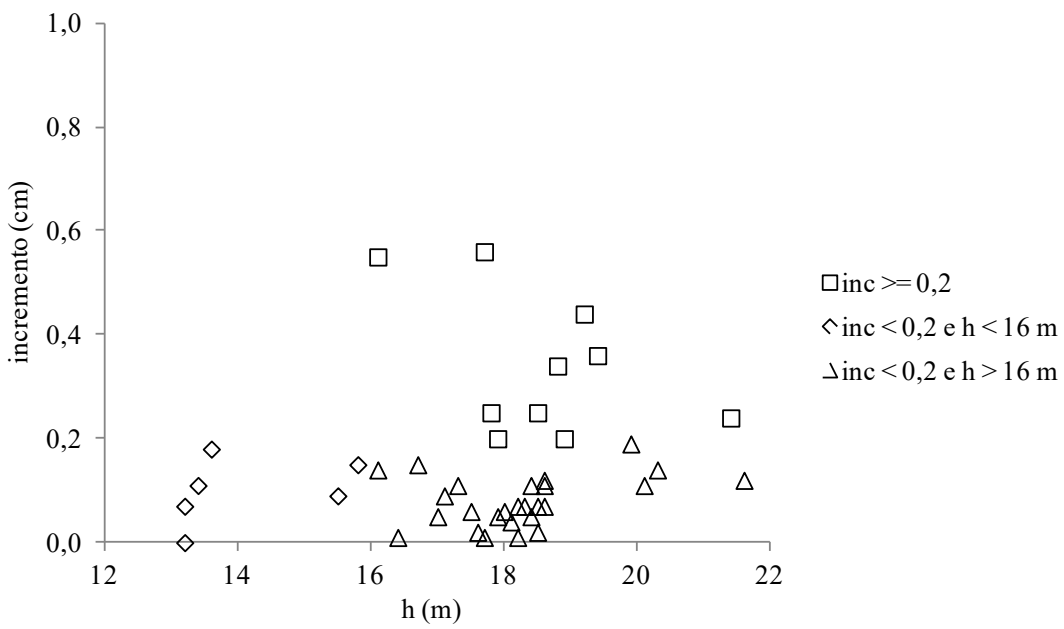

FIGURA 7: Distribuição dos incrementos em diâmetro em relação à altura para os indivíduos de Araucaria angustifolia com diâmetro entre 20 e $30 \mathrm{~cm}$.

FIGURE 7: Distribution of increments in diameter in relation to the height of Araucaria angustifolia individuals with diameters between 20 and $30 \mathrm{~cm}$. 
Os indivíduos com dap entre 20 e $30 \mathrm{~cm}$ apresentaram crescimento considerável a partir da altura mínima de $16 \mathrm{~m}$. Já para os indivíduos da classe de dap 1 (dap menor que $20 \mathrm{~cm}$ ) esta altura foi de $14 \mathrm{~m}$.

No entanto, também puderam ser observados indivíduos de araucária com altura superior a $16 \mathrm{~m}$, mas com incremento inferior a $0,2 \mathrm{~cm} /$ ano, ou seja, com crescimento reduzido.

Estes indivíduos mostraram-se em situações de alta competição, considerando a porcentagem de araucárias circundantes (Figura 8B). Todos, exceto dois indivíduos, encontraram-se em condições em que mais de $40 \%$ das árvores circundantes foram araucárias.

Além disso, a maioria desses indivíduos apresentou altos valores de densidade de Spurr, o que poderia limitar o crescimento desses indivíduos de araucária.

Os indivíduos de araucária com crescimento superior a $0,2 \mathrm{~cm} /$ ano (Figura $8 \mathrm{~A}$ ) também apresentaram altos valores da densidade de Spurr, inclusive, com valores maiores que os indivíduos com crescimento desprezível. Entendendo-se, portanto, que, para estes indivíduos, a altura foi o fator determinante para o crescimento.

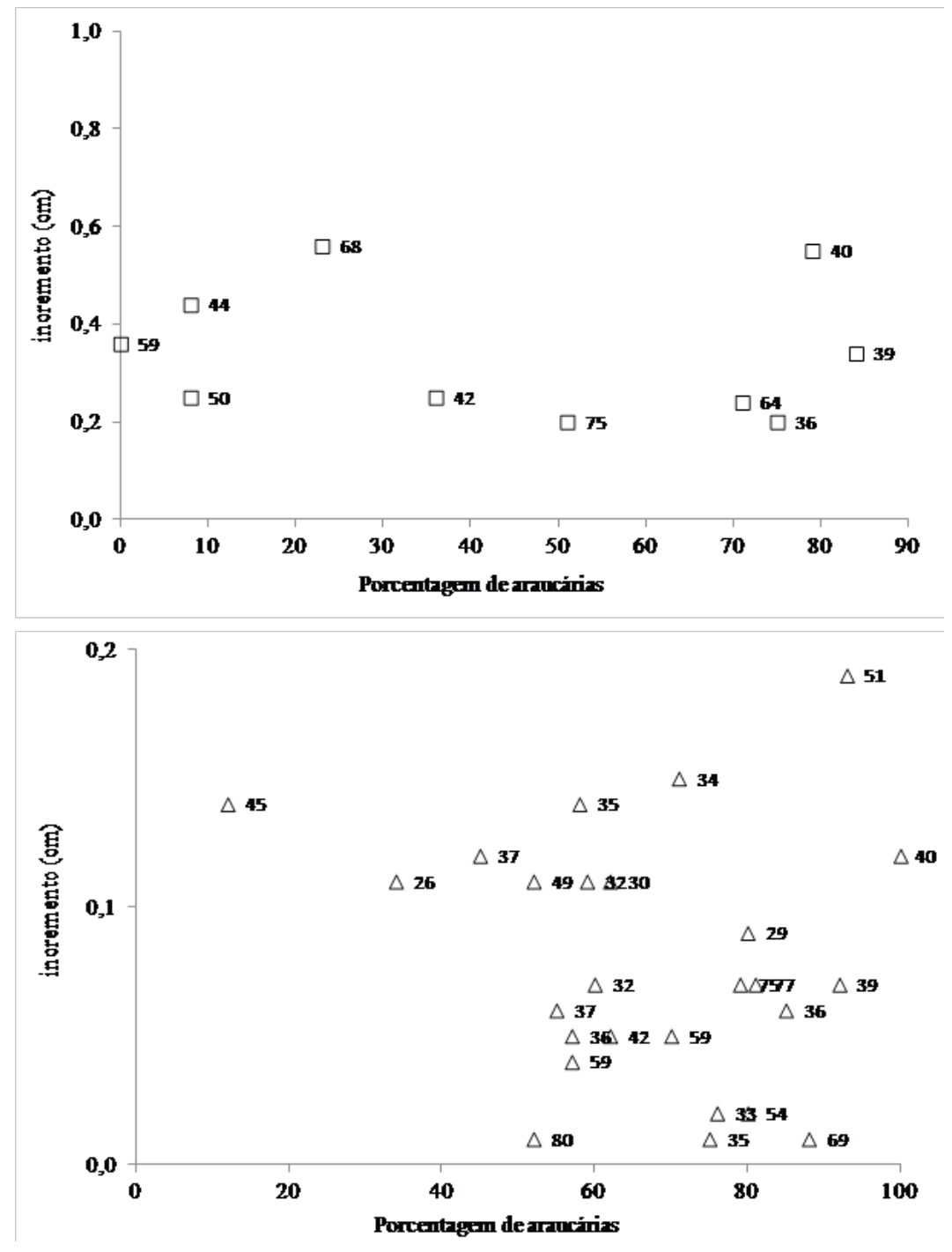

FIGURA 8: Distribuição dos incrementos em diâmetro em relação à porcentagem de araucárias concorrentes para os indivíduos de Araucaria angustifolia da classe de diâmetro 2 (Obs: os valores do rótulo de dados representam a densidade pontual de Spurr).

FIGURE 8: Distribution of increments in diameter compared the percentage of competitors araucarias for individuals of Araucaria angustifolia of the diameter class 2. 


\section{Demais classes de diâmetro}

A distribuição dos incrementos em diâmetro em relação ao dap para as classes de diâmetro 3, 4, 5, 6, 7 e 8 para os indivíduos de Araucaria angustifolia não diferiu da distribuição das classes de dap anteriores, sendo novamente observado qualquer valor de incremento para cada um dos valores de diâmetro.

Da mesma forma, indivíduos com crescimento reduzido puderam ser observados em todos os valores de diâmetro.

Para estas classes, a altura, a densidade de Spurr e a porcentagem de araucárias concorrentes não foram variáveis explicativas do incremento. Os indivíduos com crescimento inferior a $0,2 \mathrm{~cm} / \mathrm{ano}$ apresentaram valores de altura semelhantes aos indivíduos com crescimento maior que $0,2 \mathrm{~cm} / \mathrm{ano}$. Já os valores da porcentagem de araucárias concorrentes variaram de 0 a $100 \%$, sem distinção para ambos os valores de incremento.

\section{CONCLUSÕES}

Os modelos de incremento em diâmetro para árvores individuais de Araucaria angustifolia apresentaram bom ajuste em termos de coeficiente de determinação, no entanto, os resíduos foram altos e fortemente tendenciosos, sendo a sua utilização para a predição do incremento não adequada.

\section{REFERÊNCIAS}

EBLING, A. A. et al. Projeção diamétrica de Araucaria angustifolia (Bertol.) Kuntze em remanescente de Floresta Ombrófila Mista. In: CONGRESSO FLORESTAL PARANAENSE, 4., 2012. Anais... Curitiba: Congresso Florestal Paranaense, 2012.

LORENZI, H. Árvores Brasileiras: manual de identificação e cultivo de plantas arbóreas nativas do Brasil. 2. ed. Nova Odessa: Editora Plantarum, 1998. v. 2. 352 p.

REITZ, R.; KLEIN, R. M.; REIS, A. Projeto madeira do Rio Grande do Sul. Itajaí: Sellowia, 1983. 525 p.

SCOLFORO, J. R. S. et al. Modelo de produção para floresta nativa com base para manejo sustentado. Cerne, Lavras, v. 2, n. 1, 1996.

STEPKA, T. F. et al. Modelagem do increment em diâmetro em diâmetro da Araucaria angustifolia em uma Floresta Ombrófila Mista no centro-sul do Paraná. Floresta, Curitiba, v. 42, n. 3, p. 607-620, jul./set. 2012. VANCLAY, J. K. Aggregating tree species to develop diameter increment equations for tropical rainforest. Forest Ecology and Management, Amsterdam, v. 42, p. 143-168, 1991.

VANCLAY, J. K. Modelling forest growth and yield: applications to mixed tropical forest. Wallingford: CAB International, 1994. $312 \mathrm{p}$.

ZEIDE, B. Accuracyof equations describing diameter growth. Canadian Journal of Forest Research, Ottawa, v. 19, n. 10, p. 1283-1286, 1989. 